\title{
Los Entornos Virtuales de Enseñanza y Aprendizaje en la Universidad Nacional de La Plata. Una aproximación a los usos y opiniones de los estudiantes
}

\author{
The Virtual Environments of Education and Learning in the National \\ University of La Plata. An approximation to the uses and opinions of the \\ students
}

\author{
Cecilia Inés Nóbile ${ }^{1}$, Álvaro Enrique Luna ${ }^{2}$ \\ ${ }^{1}$ Facultad de Ciencias Económicas, Universidad Nacional de La Plata, Argentina (cecilia.nobile@econo.unlp.edu.ar) \\ ${ }^{2}$ Facultad de Ciencias Económicas, Universidad Nacional de La Plata, Argentina (alvaro.luna@econo.unlp.edu.ar)
}

\section{RESUMEN:}

En los últimos años el desarrollo de las Tecnologías de la Información y Comunicación han ofrecido un abanico de herramientas con un potencial muy importante para contribuir a la labor docente. Más allá de las posibilidades de realizar cursos y carreras de grado y posgrado a distancia, la educación tradicional, típicamente presencial, también se ha visto modificada por ello.

Por tanto, este trabajo tiene como objetivo general lograr una primera aproximación a la percepción de los estudiantes de la UNLP sobre el uso de los Entornos Virtuales de Enseñanza y Aprendizaje, como contribución a la calidad educativa.

El marco general del presente trabajo es el proyecto "La calidad de la educación superior. Un estudio sobre la percepción de los Estudiantes", el cual se encuentra acreditado en el Programa de Incentivos impulsado por el Ministerio de Educación, Ciencia y Tecnología de la Nación.

La metodología utilizada se corresponde con la aplicada en el mencionado proyecto siendo la información presentada aquí una parte de la relevada. Dicha metodología es de carácter descriptiva, tratándose de una investigación no experimental con un diseño transversal en la cual las fuentes de información básica utilizadas son de tipo primaria, recabada por medio de cuestionarios auto-administrados.
La información aquí presentada permite plantear la necesidad de desarrollar mayores investigaciones que aborden aspectos tales como opiniones y acciones de docentes y estudiantes para dar cuenta cuál es la contribución de las Tecnologías de la Información y Comunicación a la calidad educativa y cuál es el rol que cumplen los docentes en este proceso.

$\begin{array}{lcr}\text { PALABRAS } & \text { CLAVE: } & \text { ENTORNOS } \\ \text { VIRTUALES, } & \text { PERCEPCIÓN } & \text { DE } \\ \text { ESTUDIANTES, } & \text { EDUCACIÓN } & \text { SUPERIOR, } \\ \text { CALIDAD EDUCATIVA. } & & \end{array}$

\section{ABSTRACT:}

In recent years the development of the Technologies of the Information and Communication have offered a range of tools with high potential to contribute to the educational labor. Beyond the possibilities of taking courses and undergraduate and post-grad degrees at distance, the traditional education, typically faceto-face, has also been modified by it.

Therefore, this work has as overall goal achieve an approximation to the perception of the students of the UNLP on the use of the Virtual Environments of Education and Learning, as contribution to the educational quality. 
The general frame of the present work is the project "The quality of the higher education. A study on the perception of the Students", which is accredited in the Program of Incentives stimulated by the Department of Education, Science and Technology of the Nation.

The methodology used corresponds to that applied in the mentioned project being the information presented here part of the surveyed. This methodology is descriptive in nature, being a nonexperimental research with a transverse design in which basic information sources used are of primary type, obtained by means of selfadministered questionnaires.

The information presented here allows us to suggest the need to develop more research that address issues such as opinions and actions of teachers and students to realize which is the contribution of the Technologies of the Information and Communication to the educational quality and what is the role that the teachers fulfill in this process.

\section{KEYWORDS: VIRTUAL ENVIRONMENT, STUDENT PERCEPTION, HIGHER EDUCATION, EDUCATION QUALITY.}

\section{FUNDAMENTACIÓN Y OBJETIVOS}

En los últimos años el desarrollo de las Tecnologías de la Información y Comunicación han ofrecido un abanico de herramientas con un potencial muy importante para contribuir a la labor docente. Más allá de las posibilidades de realizar cursos y carreras de grado y posgrado a distancia, la educación tradicional, típicamente presencial, también se ha visto modificada por ello.

La Universidad Nacional de La Plata (UNLP), desde el Departamento de Educación a Distancia, Innovación en el Aula y TIC, promueve la incorporación estas nuevas herramientas para contribuir a una educación de calidad a través de charlas de sensibilización, capacitaciones y cursos específicos, asesoramiento y acompañamiento en la implementación de innovaciones en el aula, entre otras actividades.

Una de las herramientas que se encuentran a disposición de los docentes son los entornos virtuales de enseñanza y aprendizaje (EVEA), cuyas posibilidades de utilización son muchas y muy variadas. La UNLP ofrece a sus docentes y alumnos tres EVEA:

- WebUNLP, entorno virtual de enseñanza y aprendizaje, en el que docentes y alumnos comparten un espacio de trabajo, de comunicación, interacción, y acceso a recursos de interés.

- WAC o Web de apoyo a Cátedras Presenciales, permite de manera sencilla el manejo administrativo, de contenidos y de comunicación dentro de una cátedra a través de la Web.

- Aula Cavila para cursos de Posgrado, propicia el desarrollo de la investigación y la docencia al más alto nivel, aprovechando las excelencias académicas e investigadoras de cada uno de los centros implicados.

Asimismo, cada Unidad Académica tiene libertad para adoptar cualquier otro EVEA que considere adecuado, por lo que pueden existir diferencias tanto en la herramienta seleccionada como en el uso por parte de docentes y estudiantes.

Por tanto, este trabajo tiene como objetivo general lograr una primera aproximación a la percepción de los estudiantes de la UNLP sobre el uso de los EVEA, como contribución a la calidad educativa.

\section{EDUCACIÓN Y TIC}

\subsection{Las TIC en el escenario actual}

El grado de avance del conocimiento científico y tecnológico de las últimas décadas, ha posibilitado, entre otros fenómenos, el surgimiento de un "tercer entorno" (Echeverría, 1999) es decir, un nuevo espacio social con características que le son propias y que son muy diferentes a las de los entornos en los que hemos vivido los seres humanos, los naturales y urbanos. La principal diferencia que describe el autor citado es que dentro de este "tercer entorno" las personas pueden generar relaciones, interactuar, a distancia, es decir, sin coincidir en tiempo y espacio.

Esta posibilidad de interacción a distancia ha impactado, en mayor o menor medida, en todas las actividades humanas y sociales, y en particular sobre la educación, aspecto que es de especial interés en este trabajo.

Podría suponerse que el desarrollo de las tecnologías de la información y comunicación (TIC) permitió el surgimiento de diferentes tipos de cursos y carreras a distancia. Sin embargo, el concepto de educación a distancia puede remontarse hacia muchos años atrás ${ }^{1}$, ya que

\footnotetext{
${ }^{1}$ Al respecto, García Vega (2003) plantea el surgimiento de la educación a distancia en el año 1728, con la publicación en la
} 
existieron ofertas educativas que utilizaban medios como el televisor, el correo postal o el teléfono para que el alumno accediera a situaciones educativas, y materiales de estudio. La desventaja de estas propuestas, tal como señala García Aretio (2007) residía en que los canales de comunicación y recursos aplicados permitían una escasa interacción, y por ello, esta modalidad era considerada de una calidad inferior a la educación tradicional, cara a cara.

Es por ello, que el desarrollo de las (TIC) ha permitido una nueva perspectiva del proceso de enseñanza y aprendizaje aunque, según el autor citado, no introducen nuevos principios educativos $^{2}$, sino que los vuelven más fácilmente alcanzables.

Esto sería posible si consideramos a las TIC como un posible instrumento de mediación, entendiendo por mediador, en el contexto de la educación a distancia, a las propuestas humanas y no humanas de comunicación que permiten a una persona, un grupo o una organización desempeñar, total o parcialmente, las funciones de andamiaje, ayuda y negociación de sentidos en diversos soportes para que la comunicación a distancia se materialice como una práctica cultural (Fainholc, 2008). Es importante destacar que el concepto de instrumento mediador surge en el marco de la psicología del desarrollo, y desde los aportes teóricos de Lev Vigotsky, se considera que la cognición humana es posible gracias a la mediación, es decir, a instrumentos simbólicos y físicos, como el lenguaje o la escritura, que la cultura proporciona al sujeto y que le permiten mediar su relación con el mundo, con los hombres y consigo mismo (Suárez Guerrero, 2003).

\subsection{Los entornos virtuales de enseñanza y aprendizaje}

\subsubsection{Una aproximación al concepto}

Pueden hallarse numerosas definiciones acerca del concepto de entorno virtual de enseñanza y aprendizaje (EVEA), por ejemplo, en términos generales puede decirse que es una aplicación informática desarrollada con fines pedagógicos, es decir, persigue un fin educativo (Ferreira y Sanz, 2009). Del mismo modo, si ahondamos en la

Gaceta de Boston de un ofrecimiento de un material auto instructivo y de tutorías por correspondencia.

${ }^{2}$ En la obra citada, García Aretio describe los principios educativos tales como apertura, flexibilidad, democratización, socialización e interactividad, actividad, individualización y motivación. funcionalidad que posee puede describirse como el escenario físico donde un alumno o comunidad de alumnos desarrollan su trabajo, incluyendo todas las herramientas, documentos y otros artefactos que pueden ser encontrados en dichos escenarios, pero también las características socio/culturales para tal trabajo (Salinas Ibañez, 2004). Una definición interesante es la dada por Sigalés, quien plantea que los entornos virtuales son espacios de comunicación que permiten el intercambio de información y que harían posible, según su utilización, la creación de un contexto de enseñanza y aprendizaje en el que se facilitara la cooperación de profesores y estudiantes, en un marco de interacción dinámica, a través de unos contenidos culturalmente seleccionados y materializados mediante la representación, mediante diversos lenguajes que el medio tecnológico es capaz de soportar (Sigalez, 2002, citado por García Aretio, 2006).

En esta definición debe resaltarse el sentido potencial con que se describen sus funcionalidades, ya que los resultados que pueden lograrse dependerán del uso que se haga del mismo, en concordancia con unos objetivos preestablecidos.

En muchas universidades tanto públicas como privadas se ha incorporado el uso de un EVEA, tanto como apoyo de cursos presenciales como plataforma para cursos a distancia. Acorde a las políticas establecidas por cada universidad, puede citarse la clasificación de las iniciativas, expuesta por Sangrá (2001), la cual no es exhaustiva:

- Universidades presenciales que introducen elementos de virtualidad en su dinámica educativa

- Universidades presenciales con extensión universitaria virtual

- Espacios compartidos de cursos virtuales que ofrecen las universidades presenciales

- Universidades virtuales adosadas a la universidad tradicional

- Universidades virtuales como organizaciones virtuales

- Espacios virtuales interuniversitarios comunes

Esta clasificación constituye un ejemplo de la variedad de propuestas existentes dentro del ámbito de la educación superior, lo que deja entrever la complejidad que involucra analizar el uso de los EVEA en este tipo de instituciones.

En este sentido, pueden citarse numerosos estudios de caso que intentan indagar la influencia de un EVEA sobre diferentes aspectos de la 
práctica docente universitaria, pero la mayoría están referidos a cursos a distancia ya que es el tipo de propuesta donde, puede suponerse, un EVEA tendrá mayor influencia. Sin embargo, pocos estudios ahondan en el caso de cursos presenciales que se extienden más allá del aula física a partir del uso de un EVEA.

\subsection{2. ¿Implementar un EVEA en carreras presenciales?}

Los EVEA cumplen un rol fundamental en la mayoría de los cursos no presenciales que se dictan en la actualidad, ya que constituyen el medio a través del cual se logra la interacción entre docentes y alumnos y entre alumnos y materiales.

Sin embargo, que la carrera universitaria se dicte bajo una modalidad presencial no supone, en principio, que el uso de un EVEA no se posible o que no sea de utilidad.

Una de las ventajas básicas que trae consigo la utilización de este tipo de entornos es la integración de diferentes herramientas y servicios para gestionar y desarrollar actividades de formación (Ferreira y Sanz, 2009). Por otro lado, pueden citarse algunas características de los EVEA, mencionadas por González, Esnaola y Martín en su libro Propuestas Educativas mediadas por tecnologías digitales: Algunas pautas de trabajo:

- Promociona y facilita la comunicación entre profesorado, tutores y alumnos.

- El trabajo grupal se combina con el individual.

- Ofrece recursos para el acceso y difusión de la información on-line.

- Ofrece herramientas de gestión para las grandes instituciones.

Tal como sintetizan Ferreira y Sanz (2009):

"Los EVEA sirven para distribuir materiales educativos en formato digital (textos, imágenes, audio, simulaciones, juegos, etc.) y acceder a ellos, para realizar debates y discusiones en línea sobre aspectos del programa de la asignatura, para integrar contenidos relevantes de la red o para posibilitar la participación de expertos o profesionales externos en los debates o charlas" (p. 11)

Estas características no resultan propias de la educación a distancia, sino que podrían aplicarse al uso de un EVEA como complemento de un curso presencial. En realidad, las ventajas de la aplicación de este tipo de herramientas dependen, en gran medida, de los conocimientos y utilidades que le den los docentes.
En trabajos anteriores (Nóbile y Luna, 2013) se presentaron algunos interrogantes sobre el proceso de implementación de los EVEA, dada la información obtenida de proyectos de investigación sobre las percepciones de los docentes universitarios argentinos.

En uno de los apartados de la encuesta realizada por la Red ECUALE $^{3}$ en el proyecto DOCALE $^{4}$, se instaba a los docentes a responder lo siguiente: "Evalúe la IMPORTANCIA que cree que tienen sobre la MEJORA DE LA CALIDAD de la educación universitaria los siguientes factores y el grado en que considera que cada uno de los mismos se encuentra PRESENTE EN SU FACULTAD. (Califique cada punto del 1 al 5 . Considere 1 como muy bajo/nulo y 5 como muy alto)".

El factor que aquí nos interesa es "Utilización de plataformas tecnológicas de apoyo a la docencia (campus virtual o plataformas similares)". Si se observa este factor frente a los otros factores listados, la importancia dada fue escasa. Asimismo, analizando en detalle los datos obtenidos, se llegaron a algunas conclusiones interesantes, ya que se planteó que si bien se reconocía, en cierta medida, la importancia que tiene el uso de esta herramienta en la educación superior, los docentes no percibían su existencia en sus universidades. Es importante resaltar que las respuestas obtenidas de los docentes pertenecientes a la UNLP siguen la tendencia general.

\section{LOS EVEA EN LA UNIVERSIDAD NACIONAL DE LA PLATA}

\subsection{Aspectos metodológicos}

El marco general del presente trabajo es el proyecto "La calidad de la educación superior. Un estudio sobre la percepción de los Estudiantes", el cual se encuentra acreditado en el Programa de Incentivos impulsado por el Ministerio de Educación, Ciencia y Tecnología de la Nación.

La metodología utilizada se corresponde con la aplicada en el mencionado proyecto siendo la información presentada aquí una parte de la

\footnotetext{
${ }^{3}$ La Red de Estudios sobre la Calidad en las Universidades de América Latina y España (ECUALE), fue formada en el 2007 por docentes de la Universidad del País Vasco/EuskalHerrikoUnibertsitatea (España), la Universidad de Guadalajara (México) y la Universidad Nacional de La Plata (Argentina).

${ }^{4} \mathrm{La}$ sigla hace referencia al título "Determinantes y orientaciones de la calidad de la educación superior en América Latina y España".
} 
relevada. Dicha metodología es de carácter descriptiva, tratándose de una investigación no experimental con un diseño transversal en la cual las fuentes de información básica utilizadas son de tipo primaria, recabada por medio de cuestionarios auto-administrados.

Es importante aclarar que al momento no se encuentran sistematizadas la totalidad de las encuestas realizadas ${ }^{5}$, restando el $30 \%$ de las mismas. Se cree que los datos analizados hasta ahora resultan sumamente útiles y permiten la elaboración de conclusiones que, aunque preliminares, contribuyen a comprender la realidad estudiada.

\subsection{Presentación de los datos}

$\mathrm{Si}$ recordamos la clasificación de Sangrá mencionada anteriormente, la UNLP podría ubicarse en la categoría "Universidades presenciales que introducen elementos de virtualidad en su dinámica educativa", ya que la modalidad de dictado de sus carreras de grado es fundamentalmente la presencial. Sin embargo hay innumerables experiencias de incorporación de tecnología informática en los procesos de enseñanza y aprendizaje, así como cursos de formación para docentes cuyo dictado es a distancia.

En este marco, se presentan los datos obtenidos. La encuesta utilizada instaba a los alumnos a indicar cierto grado de importancia a una serie de factores que pueden contribuir a la calidad educativa. Luego, a que indiquen el grado en que considera que cada uno de los mismos se encuentra presente en su facultad.

De acuerdo a los datos relevados, el 59\% de los alumnos asignaron un grado de importancia "Alta" al uso de plataformas tecnológicas en apoyo a la docencia como factor para mejorar la calidad educativa y un porcentaje semejante percibe que en su facultad se le da una importancia similar, tal como se muestra en el Cuadro 1. Si nos concentramos en las personas que le dan una importancia baja, se puede afirmar que solo un $7 \%$ del total lo considera así.

\footnotetext{
${ }^{5} \mathrm{Al}$ momento, se encuentran sistematizadas y analizadas 323 encuestas.
}

Tabla 1. Utilización de plataformas tecnológicas de apoyo a la docencia (campus virtual o plataformas similares). Evalúe la importancia que cree que tienen sobre la mejora de la calidad de la educativa y el grado en que se encuentran presentes en su facultad

\begin{tabular}{ccccc}
\hline $\begin{array}{c}\text { Grado de } \\
\text { importancia }\end{array}$ & \multicolumn{2}{c}{ Importancia } & \multicolumn{2}{c}{ Presencia } \\
\hline & $\begin{array}{c}\text { Respuestas } \\
(\text { Abs })\end{array}$ & $\begin{array}{c}\text { Respuestas } \\
(\%)\end{array}$ & $\begin{array}{c}\text { Respuestas } \\
(\text { Abs })\end{array}$ & $\begin{array}{c}\text { Respuestas } \\
(\%)\end{array}$ \\
\cline { 2 - 5 } Baja & 21 & $7 \%$ & 33 & $10 \%$ \\
Media & 105 & $33 \%$ & 84 & $26 \%$ \\
Alta & 190 & $59 \%$ & 197 & $61 \%$ \\
No sabe & 7 & $2 \%$ & 9 & $3 \%$ \\
\hline TOTAL & 323 & $100 \%$ & 323 & $100 \%$ \\
\hline
\end{tabular}

Fuente: Elaboración propia

Al preguntar si se utiliza un EVEA en su facultad, el $82 \%$ respondió afirmativamente, un $15 \%$ que no se usa y un $3 \%$ no respondió. Esto puede observarse en el Cuadro 2.

Tabla 2. Uso de EVEA en su Facultad

\begin{tabular}{lll}
\hline Uso de EVEA & & TOTAL \\
\hline \multirow{3}{*}{ No } & Absol. & $\%$ \\
\cline { 2 - 3 } $\mathrm{Si}$ & 48 & $15 \%$ \\
$\mathrm{Ns} / \mathrm{Nc}$ & 265 & $82 \%$ \\
\hline TOTAL & 10 & $3 \%$ \\
\hline
\end{tabular}

Fuente: Elaboración propia

Los EVEA más utilizados según los alumnos son el Moodle (38\%) y el WebUNLP (28\%), luego, en menor medida aparece la WAC (3\%). Se observa un porcentaje de alumnos que no reconoce cuál es el EVEA que se usa en su unidad académica (28\%), aunque afirman que sí se utilizan, solo que no saben su nombre. El Cuadro 3 muestra los EVEA más utilizados en las facultades.

Tabla 3. EVEA que utiliza en su Facultad

\begin{tabular}{lll}
\hline $\begin{array}{l}\text { Entorno } \\
\text { utilizado }\end{array}$ & $\begin{array}{l}\text { Respuestas } \\
(\text { Absol })\end{array}$ & $\begin{array}{l}\text { Respuestas } \\
(\%)\end{array}$ \\
\hline Moodle & 124 & $38 \%$ \\
WebUNLP & 90 & $28 \%$ \\
WAC & 10 & $3 \%$ \\
Otro & 9 & $3 \%$ \\
Ns/Nc & 90 & $28 \%$ \\
\hline Total & 323 & $100 \%$ \\
\hline
\end{tabular}

Fuente: Elaboración propia

Finalmente, se preguntó a los alumnos qué uso le daban al EVEA, dándoles las siguientes opciones: 
1. Informarse sobre novedades publicadas por los docentes.

2. Participar en foros de debate

3. Compartir documentos con su grupo de estudio

4. Comunicarse con sus compañeros

5. Comunicarse con docentes y/o tutores.

6. Entregar actividades

7. Obtener bibliografía para estudiar

8. Obtener información básica de las materias: programa, bibliografía cronograma, etc.

9. Otro. ¿Cuál?

Figura 1. Usos de los EVEA (Frecuencia absoluta)

\section{Usos del EVEA}

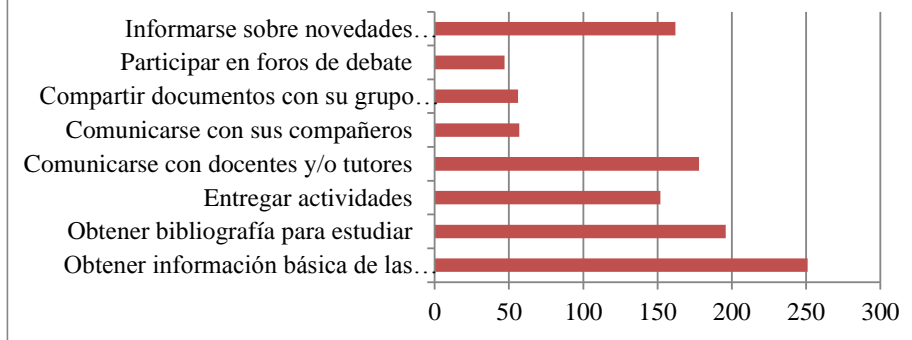

Fuente: Elaboración Propia

Los resultados obtenidos muestran que los EVEA se utilizan principalmente para obtener información sobre diferentes aspectos, siendo menor el uso de acciones que impliquen más interacción. En el Gráfico A se puede observar las frecuencias de las respuestas.

Para un análisis más detallado se han agrupado las diferentes opciones en 3 grupos:

1. Opciones relacionadas con la obtención de información $(1,2$ y 8$)$

2. Opciones relacionadas con la comunicación (4 y 5)

3. Opciones relacionadas con la realización de diferentes tipos de tareas (3, 6 y 7$)$.

Esto permite confirmar la observación realizada acerca del uso más frecuente de los EVEA, relacionado con actividades más "pasivas" o hasta tradicionales (Ver Figura 2).
Figura 2. Uso de los EVEA - Agrupados

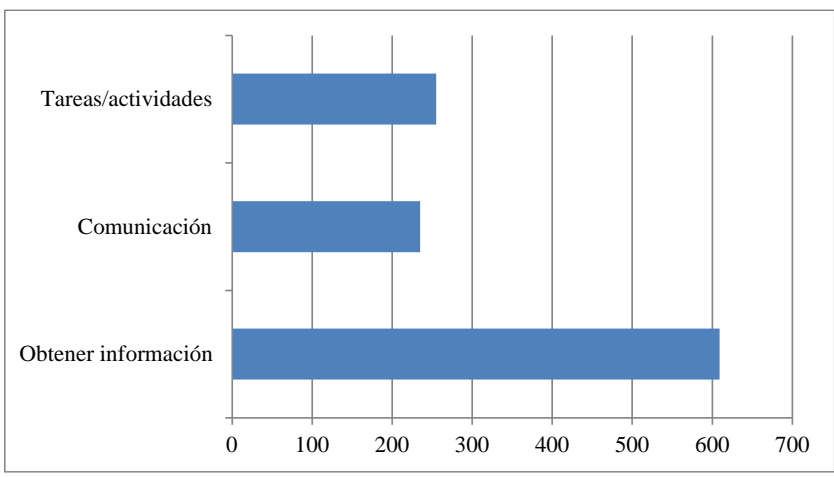

Fuente: Elaboración propia

\section{CONCLUSIONES}

Tal como se mencionó con anterioridad, los datos obtenidos no son aún definitivos, ya que la sistematización de las encuestas no ha concluido. Sin embargo, la información expuesta en el presente trabajo permite elaborar una serie de interrogantes que sirven de guía para debatir acerca de la implementación y usos de los EVEA en la Educación Superior.

En trabajos anteriores, ya citados, se abría el debate sobre cuál era la contribución real de las TIC a la calidad educativa y qué rol cumplían los docentes en el proceso de integración de las mismas a la Educación Superior. Se planteó la pregunta ¿Es importante conocer la percepción del docente sobre las posibilidades y usos de los EVEA para lograr la apropiación de este tipo de herramientas por parte de los alumnos?

En el presente trabajo se analizaron las respuestas sobre este temática de otro de los actores del proceso de enseñanza y aprendizaje, el estudiante, en particular, de la Universidad Nacional de La Plata. Se expusieron resultados que pueden llevar a conclusiones interesantes:

- Los estudiantes creen que el uso de entornos virtuales de enseñanza y aprendizaje en la Educación Superior resulta importante para mejorar la calidad educativa. La importancia asignada es mayor que la de los docentes.

- Así, perciben una fuerte existencia de este tipo de herramienta en las Facultades.

- Esto se confirma con la gran cantidad de estudiantes que afirman usar un EVEA en su Institución.

- El EVEA más utilizado es el Moodle, plataforma de acceso libre, y no los desarrollados por la UNLP. Cabría preguntarse las razones de esta elección. 
- Sin embargo, parecen no aprovecharse las ventajas principales que brinda esta herramienta, ya que sus usos principales están relacionados con la obtención de información y materiales de estudio.

Cabe preguntarse si esta última aseveración es motivada por la falta de herramientas para aprovechar este tipo de ventajas o si, estando estas disponibles, los estudiantes prefieren utilizar otros canales para la comunicación y discusión entre ellos mismos.

La información aquí presentada permite plantear la necesidad de desarrollar mayores investigaciones sobre opiniones $\mathrm{y}$ acciones de docentes y estudiantes para responder interrogantes tales como ¿Los estudiantes fueron capacitados en el uso de las TIC con fines educativos? ¿En qué instancia de su proceso formativo? ¿Cuál es el factor clave para lograr la apropiación de las TIC por parte de los estudiantes? ¿Qué rol cumplen los docentes en la mencionada apropiación? ¿Qué grado de influencia ha tenido el proceso de integración de las TIC planteado desde lo institucional? ¿El uso de los EVEA detectado responden a los objetivos de los docentes o la falta de aprovechamiento responde a la falta de capacitación? ¿Los estudiantes desearían un mayor y distinto uso de los EVEA? ¿Los estudiantes están utilizando otras plataformas no institucionales para reemplazar las funcionalidades que los EVEA no le están ofreciendo? ¿Qué rol desempeña el docente como ejemplo para el uso de los EVEA por parte de los estudiantes? ¿Los docentes estarían preparados para potenciar el uso de los EVEA en las Facultades de la UNLP?

Estas son solo algunas de las preguntas que parece interesante debatir e intentar construir las respuestas para ir formando un camino que lleve a repensar cuál es la contribución de las TIC a la calidad educativa y cuál es el rol que cumplen (o deberían cumplir) los docentes en este proceso.

\section{REFERENCIAS}

Echeverría, J. (1999). Los Señores del aire: Telépolis y el tercer entorno. Barcelona: Destino.

Fainoolc, B. (2008). Programas, profesores y estudiantes virtuales. Una sociología de la educación a distancia. Buenos Aires: Santillana.

Ferreira, A., y Sanz, C. (2009). Hacia un modelo de evaluación de entornos virtuales de enseñanza y aprendizaje. La importancia de la usabilidad. Revista Iberoamericana de Tecnología en Educación y Educación en Tecnología, 4, 10-21.
García Aretio, L. (2006). De la educación a distancia a la educación virtual. Barcelona: Ariel.

González, A., Esnaola, F., y Martín, M. (2012). Propuestas educativas mediadas por tecnologías digitales: Algunas pautas de trabajo. La Plata: Editorial de la UNLP.

Nóbile, C., y Luna, A. (2013). El uso de entornos virtuales de enseñanza y aprendizaje ¿contribuye a la calidad educativa? Una aproximación a la percepción de los profesores de universidades públicas argentinas. Congreso de Docencia Universitaria, Universidad de Buenos Aires.

Salinas Ibañez, J. (2004). Cambios metodológicos con las TIC. Estrategias didácticas y entornos virtuales de enseñanza-aprendizaje. Revista REDINED - Red de Información Educativa, Bordón Madrid, 56(3-4), 469481.

Sangrá, A. (2001). La calidad en las experiencias virtuales de educación superior, ponencia en el Encuentro Virtual Educa, organizado por la Organización de Estados Americanos (OEA). Recuperado de http://www.uoc.edu/web/esp/art/uoc/0106024/sangra. html

Suárez Guerrero, C. (2003). Los entornos virtuales de aprendizaje como instrumentos de mediación. Revista Teoría de la Educación: Educación y Cultura en la sociedad de la Información, 4. Recuperado de $\mathrm{http} / / /$ campus.usal.es/ teoriaeducacion/rev_numero_0 4/n4_art_suarez.htm 